\title{
Analogies Between Scaling in Turbulence, Field Theory and Critical Phenomena
}

\author{
Gregory Eyink and Nigel Goldenfeld \\ Physics Department and Beckman Institute, University of Illinois at Urbana-Champaign, 1110 West Green Street, Urbana, Il. \\ 61801-3080
}

\begin{abstract}
We discuss two distinct analogies between turbulence and field theory. In one analogue, the field theory has an infrared attractive renormalization-group fixed point and corresponds to critical phenomena. In the other analogue, the field theory has an ultraviolet attractive fixed point, as in quantum chromodynamics.
\end{abstract}

PACS numbers: 47.27.Ak, 64.60.Ak, 11.10.Jj

In this brief note, we shall discuss two distinct analogies between turbulence at high Reynolds number, statistical field theory and critical phenomena. Such analogies are implicit in attempts to make a useful connection between these apparently disparate phenomena, but a number of points deserve to be made explicitly. Some of our remarks are essentially trivial observations; nevertheless their ramifications have not always been respected by approximate theories [1].

The fully-developed turbulent regime is specified by the condition that the integral length-scale $L$ of the largest eddies should be much larger than the length scale $\eta_{d}$ at which dissipation is effective. The latter is usually defined as the inverse of the wavenumber $k_{d}$ at which occurs the peak of the enstrophy spectrum $k^{2} E(k)$. Equivalently, the Reynolds number $\operatorname{Re} \equiv U L / \nu$ should be large, where $U$ is a characteristic large-scale velocity and $\nu$ is the molecular viscosity. We consider the statistical steady-state with constant mean rate $\bar{\varepsilon}$ of energy injection by the turbulence production mechanism, which, by stationarity, is also the mean rate of energy dissipation by molecular viscosity. We shall take the point of view that the problem of turbulence is to understand the properties of the stationary (but presumably non-Gibbsian) probability distribution governing the velocity field of a turbulent fluid; it is this distribution which is used below when we write averages in the context of turbulence.

The first analogue of turbulence is to field theories with an infra-red attractive renormalization-group (RG) fixed point. This is the situation for field theories corresponding to critical lattice spin-systems. For example, consider a system of spins $\sigma(\mathbf{r})$ in $d$ spatial dimensions, where $\mathbf{r}$ runs over a lattice $a \mathbf{Z}^{d}$ of lattice-constant $a$, governed by a (dimensionless) Hamiltonian of the form:

$$
H[\sigma]=\sum_{\mathbf{r} \in a \mathbf{Z}^{d}} a^{d}\left[\frac{K}{2} \sum_{\mu=1}^{d}\left(\frac{\sigma\left(\mathbf{r}+a \hat{\mathbf{e}}_{\mu}\right)-\sigma(\mathbf{r})}{a}\right)^{2}+\frac{\tau}{2} \sigma^{2}(\mathbf{r})+\frac{\gamma}{4} \sigma^{4}(\mathbf{r})+\cdots\right],
$$

with $\hat{\mathbf{e}}_{\mu}, \mu=1,2, \ldots, d$ being unit lattice vectors. The parameter $\tau$ depends upon the temperature of the spin system, with an order-disorder transition occuring at the critical value $\tau_{c}$. In the critical regime $\left(\tau-\tau_{c}\right) / \tau_{c} \ll 1$ there is, for large space-separation, universality from the specific short-distance, or lattice-scale interactions. On the other hand, turbulence scaling in the high wavenumber inertial-range is believed to exhibit universality from the small wavenumber stirring mechanisms. Therefore, in this analogy the roles of space and wavenumber are interchanged. A detailed list of correspondences (and definitions) may be drawn up for an analogy along these lines, which is motivated below:

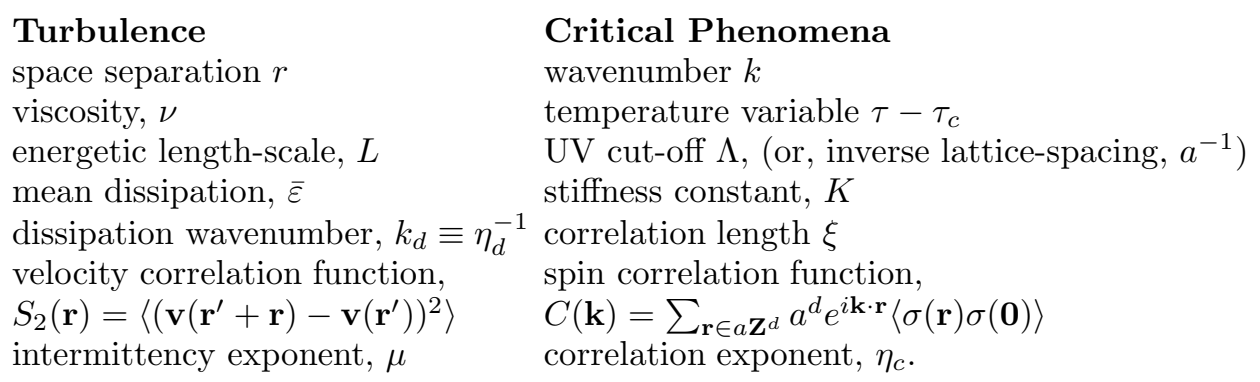

This analogue is similar to that pointed out previously by Nelkin [2], deGennes [3] and Rose and Sulem [4], the critical $\left(\tau \rightarrow \tau_{c}\right)$ limit of equilibrium spin systems being considered analogous to the zero-viscosity limit of turbulence. However, we have made a correspondence of the constants $\bar{\varepsilon}$ and $K$ which does not seem to have been pointed out 
before. Also, we propose that $\mu$ and $\eta_{c}$ are directly analogous, rather than being related as $\mu \leftrightarrow \frac{2}{3}-\eta_{c}$, as suggested by the earlier authors.

The above analogy can be motivated by comparing Kolmogorov's 1941 theory of turbulence [5] and Landau's 1937 "mean-field" theory of critical phenomena [6]. In both theories, critical exponents are obtained by dimensional analysis [7].

In the Landau mean-field analysis, it is implicitly assumed that the long wavelength properties are independent of the lattice constant $a$. Thus the limit $a \rightarrow 0$ of spin-correlations is presumed to exist, an assumption that the asymptotics are of the first kind 8 . In that case the only remaining parameter with units of length is $r_{0}^{-1 / 2}=\left(\left(\tau-\tau_{c}\right) / K\right)^{-1 / 2}$, yielding the Landau prediction for the correlation length $\xi_{L} \sim r_{0}^{-1 / 2}$. The values for the critical exponents follow from this assumption: for example,

$$
C(\mathbf{k}) \sim K \cdot k^{-2},
$$

for $k \xi \gg 1$, where it has also been assumed that the critical limit $r_{0} \rightarrow 0$ exists. In fact, Landau's first assumption that the limit $a \rightarrow 0$ exists is generally wrong, and the correct asymptotic scaling behavior is of the second kind [8]

$$
C(\mathbf{k}) \sim K \cdot k^{-2}(k a)^{\eta_{c}},
$$

for some $\eta_{c}>0$, in which the microscopic length $a$ appears explicitly. Likewise, the Landau scaling of the correlation length has an "anomalous" correction arising from $a$-dependence: $\xi \sim \xi_{L} \cdot\left(r_{0} a^{2}\right)^{\theta}$ for some $\theta>0$, or $\xi \sim r_{0}^{-\nu_{c}}$ with $\nu_{c}=\frac{1}{2}-\theta$.

In the same way, Kolmogorov assumed that the limit $L \rightarrow+\infty$ should exist in turbulence with finite velocity correlations. In that case, the only remaining length-scale is the dissipative scale estimated as $\eta_{K} \sim(\bar{\varepsilon})^{-1 / 4} \nu^{3 / 4}$. Thus, Kolmogorov obtained by dimensional analysis

$$
S_{2}(r) \sim(\bar{\varepsilon} r)^{2 / 3},
$$

when $k_{d} r \gg 1$, if the limit $\nu \rightarrow 0$ exists for velocity correlations. As with the corresponding assumption in Landau's theory, Kolmogorov's hypothesis of existence of the first $L \rightarrow+\infty$ limit is questionable, due to the build-up of fluctuations of energy flux in the energy cascade. (Ironically, this criticism originates in part from a remark of Landau himself [9]!). Instead, simple cascade models indicate that the scaling law should instead be of the form

$$
S_{2}(r) \sim(\bar{\varepsilon} r)^{2 / 3}\left(\frac{L}{r}\right)^{-\mu}
$$

for some $\mu>0$. Similarly, these models suggest [10] that the dissipation scale may have a slight dependence upon $L$, as $\eta_{d} \sim \eta_{K}\left(\frac{L}{\eta_{K}}\right)^{\delta}$ for $\delta \neq 0$, leading to a scaling $\eta_{d} \sim \eta_{K}^{\omega}$ with $\omega=1-\delta$.

It is clear that in these formulas, $K, a, \eta_{c}, \nu_{c}$ are homologous, respectively, to $\bar{\varepsilon}, L^{-1}, \mu, \omega$. Notice that typically $\eta_{c}$ is small in critical systems even in two or three dimensions and that $\mu$ represents an empirically small modification to the dimensional analysis result of $5 / 3$ for the exponent appearing in the Fourier transform of $S_{2}$ :

$$
E(k) \sim(\bar{\varepsilon})^{2 / 3} k^{-5 / 3}(k L)^{-\mu} .
$$

The constants $K$ and $\bar{\varepsilon}$ play a similar formal role in the two theories. To see this, consider a Martin-Siggia-Rose (MSR) Lagrangian 11.12 for the steady-state turbulent cascade state produced by driving with a Gaussian random force $\mathbf{f}$, white noise in time, with zero mean and covariance

$$
\left\langle f_{i}(\mathbf{r}, t) f_{j}\left(\mathbf{r}^{\prime}, t^{\prime}\right)\right\rangle=2 \delta_{i j} F\left(\mathbf{r}-\mathbf{r}^{\prime}\right) \delta\left(t-t^{\prime}\right) .
$$

It is easy to check that $F$ has the units of energy dissipation and, indeed, it is directly related to the mean energy injection rate by $\bar{\varepsilon}=2 F(0)$. Therefore, the quadratic term in the MSR action corresponding to the "response field" $\hat{\mathbf{v}}$ is

$$
S^{(0)}[\hat{\mathbf{v}}]=\frac{1}{2} \int d t \int d \mathbf{r} d \mathbf{r}^{\prime} \hat{\mathbf{v}}(\mathbf{r}, t) F\left(\mathbf{r}-\mathbf{r}^{\prime}\right) \hat{\mathbf{v}}\left(\mathbf{r}^{\prime}, t\right),
$$

which is proportional to $\bar{\varepsilon}$. $S^{(0)}$ has a formally similar structure to the quadratic term in the spin-Hamiltonian, which is proportional to $K$. 
A second, rather different analogy can be made between turbulence and field theory with an ultraviolet (UV) attractive fixed point, or, field theory for short. (It is generally accepted that, in order for a continuum field theory model to be well-defined, it must be "asymptotically safe," i.e. it must correspond to a lattice model with a UV attracting fixed point.) Field theory, such as UV asymptotically free quantum chromodynamics (QCD), exhibits scaling at short distances, just as turbulence is believed to do. Therefore, in this analogy space corresponds to space. The spin system we examined before may still be used in this analogy, when it is considered in dimension $d<4$ along the single RG trajectory which flows in the UV direction into the non-Gaussian Wilson-Fisher fixed point (previously we considered the theory at a generic point on or slightly away from the critical surface). It is convenient to use the field-theoretic notation, introducing a (lattice) field

$$
\phi(\mathbf{r})=K^{1 / 2} \sigma(\mathbf{r}),
$$

which has dimension $\frac{1}{2}(d-2)$ in units of inverse-length. As before, a detailed list of correspondences may be drawn up:

\section{Turbulence}

space separation $r$

viscosity $\nu$ (or, Kolmogorov scale, $\eta_{K}$ )

energetic length-scale, $L$

Kolmogorov wavenumber, $k_{\eta}$

velocity correlation function,

$S_{2}(\mathbf{r})=\left\langle\left(\mathbf{v}\left(\mathbf{r}^{\prime}+\mathbf{r}\right)-\mathbf{v}\left(\mathbf{r}^{\prime}\right)\right)^{2}\right\rangle$
Field Theory

space separation $r$

lattice-spacing $a$

correlation length $\xi$

UV cut-off $\Lambda=a^{-1}$

field-theoretic Green function,

$G(\mathbf{r})=\langle\phi(\mathbf{r}) \phi(\mathbf{0})\rangle$

These correspondences essentially all follow from the comparison in the last line. For example, the length-scale $L$ gets its name "integral-scale" from the fact that it is an integral correlation length of the longitudinal velocity correlation $f(r)=\left\langle\left(\hat{\mathbf{r}} \cdot\left[\mathbf{v}\left(\mathbf{r}^{\prime}+\mathbf{r}\right)-\mathbf{v}\left(\mathbf{r}^{\prime}\right)\right]\right)^{2}\right\rangle$, defined by

$$
L=\frac{1}{f(0)} \int_{0}^{\infty} d r f(r),
$$

Therefore, it corresponds naturally to the correlation length $\xi$ defined by the exponential decay rate of $G(\mathbf{r})$. Likewise, the dissipation length $\eta_{K}$ provides a short-distance cut-off for turbulent velocity correlations, analogous to the lattice cutoff $a$ for the field theory correlations. The correspondences made here, $\left(k_{\eta}, L\right) \leftrightarrow(\Lambda, \xi)$, are the opposite of those made in the first analogy, $\left(k_{\eta}, L\right) \leftrightarrow(\xi, \Lambda)$. In the present analogy, the zero-viscosity limit is analogous to the continuum limit $a \rightarrow 0$ of field theory.

Having pointed out the analogies between turbulence and field theory, it is appropriate to emphasize certain important distinctions. First, we discuss the issue of universality. In turbulence, short-distance scaling is believed to be generic and essentially independent of large-scale statistics or driving mechanisms. Scaling laws, strictly speaking, require an inertial range of infinite extent, so that we confine our discussion here to the idealized situation of zero molecular viscosity (i.e. the critical limit according to our first analogy). We will assume that the stationary probability distribution governing the turbulent state is a fixed point in a function space, whose axes may be thought of as coupling constants for all conceivable local (and perhaps non-local) operators. This distribution is usually assumed to be the fixed point of a RG transformation which integrates out short wavelength degrees of freedom, just as in critical phenomena. (An alternative to this procedure will be mentioned below.) Short-distance universality implies that the ultra-violet RG flows should all be in towards the fixed point: this distribution is a global sink in the ultra-violet. (Here again we ignore crossover phenomena associated with finite molecular viscosity or other short-distance regularization mechanisms.) Thus, the usual infra-red RG flow diagram, moving the system to longer and longer length scales, will contain a fixed point with a presumably infinite number of relevant directions. The physical significance of relevant directions, in the context of critical phenomena, is that they represent the parameters that must be tuned in order for the system to be at criticality (e.g. temperature is at the critical temperature, external field is zero). In the context of turbulence, these relevant directions correspond to the myriad of different large-scale stirring mechanisms which generate the same short-distant behaviour. One of these relevant directions, corresponding to the temperature, is the "eddy viscosity", which is the effective viscosity at a given scale generated by turbulent degrees of freedom at shorter scales. The eddy viscosity will tend to the bare, molecular viscosity (assumed zero) as the turbulence fixed-point is approached at short length-scales. In contrast, even field-theories with a UV fixed point must generally be tuned to lie on the low-dimensional surface which attracts to the fixed point, and as mentioned above, the number of variables that must be tuned is equal to the number of IR unstable directions at the fixed point: this is usually a finite number. This general argument does not distinguish between relevant and marginally relevant directions. 
The second distinction is that scaling behavior may be quite different in turbulence and typical field-theories. The latter usually show simple "gap-scaling " or "hyperscaling" in which higher-order correlations scale with exponents which are just integer multiples of a single field dimension. For example, if $d_{\phi}$ is the scaling dimension of the field $\phi$, then typically

$$
\left\langle\phi\left(\lambda \mathbf{r}_{1}\right) \cdots \phi\left(\lambda \mathbf{r}_{p}\right)\right\rangle \sim \lambda^{-p \cdot d_{\phi}}, \quad p=1,2,3, \ldots
$$

as $\lambda \rightarrow 0$. In contrast, higher-order velocity structure functions, $S_{p}(r)$, are believed to scale in high Reynolds number turbulence with $r \rightarrow 0$ as

$$
\left\langle\left(\hat{\mathbf{r}} \cdot\left[\mathbf{v}\left(\mathbf{r}^{\prime}+\mathbf{r}\right)-\mathbf{v}\left(\mathbf{r}^{\prime}\right)\right]\right)^{p}\right\rangle \sim(\bar{\varepsilon} r)^{p / 3}\left(\frac{L}{r}\right)^{-x_{p}},
$$

for $x_{p}<0$ 13]. This type of "multifractal" scaling is found in very few field theories. In the cases where it occurs, it is usually associated with sequences of field variables with negative scaling dimensions. More generally, the multifractal scaling occurs if there is a sequence of variables "additively coupled" in the operator product expansion and negative scaling dimensions are one common mechanism by which such sequences are produced [15, 16]. A representative case is the $O(N)$ nonlinear $\sigma$-model in dimension $d=2+\varepsilon$ with $N<2$ [17], which has such a sequence. This example is closely related to Wegner's theory of the mobility edge in disordered electron systems and the hypothesis there of multifractal electron wavefunctions for the localized states [17. Note that any variable with scaling dimension $x<d$ corresponds to an unstable direction at the fixed point. Therefore, the variables with negative scaling dimensions correspond to an infinite set of unstable directions, and the associated fixed point is UV attractive in a large domain, as suggested in the preceding paragraph. Motivated by this type of example, an RG theory of high Reynolds number scaling in turbulence can be developed [19,20] in which the "anomalous dimensions" are the negative scaling dimensions $x_{p}$ of the powers of the velocity-gradients, $(\nabla \mathbf{v})^{p}$. In traditional turbulence terminology this corresponds to generalized flatnesses for velocity gradients diverging as powers of the Reynolds number, which is also observed in both simulation and experiment 16,21. The same phenomena lead also to the possibility of a hierarchy of dissipation lengths $\eta_{d}^{(p)}$ delimiting the short-distance end of the scaling range of the $p$ th structure functions $S_{p}$, each having a different dependence on molecular viscosity $\eta_{d}^{(p)} \sim \eta_{K}^{\omega_{(p)}}$ [10.

The third distinction that we wish to mention is the form of the continuum limit. In field theory taking the continuum limit requires making a multiplicative renormalization of the lattice field-variables,

$$
[\phi](\mathbf{r})=Z(a) \cdot \phi(\mathbf{r}),
$$

where $Z(a) \sim a^{-\gamma_{\phi}}$ and $\gamma_{\phi}$ is an "anomalous dimension" of the renormalized field $[\phi]$. The necessity of this renormalization is connected to the UV divergences in the field-theoretic Greens functions of the "bare" fields $\phi$ (cf. Eq.(3)). Unlike the fields $[\phi]$ which exist only as distributions in the limit $a \rightarrow 0$, the velocity field in turbulence must remain an ordinary function in the limit $\nu \rightarrow 0$. The requirement of finite mean kinetic energy $\left\langle\frac{1}{2} v^{2}\right\rangle\langle\infty$ implies this (since the velocity field must then be locally square-integrable with probability one). On the other hand, the velocity gradients will exist necessarily only as distributions and UV divergences may appear associated to their products at a single space-point. Only these products require "renormalization" (by suitable powers of $\eta_{K}$ ) in the zero-viscosity limit and any "anomalous dimensions" in inertial-range turbulence scaling must therefore be associated with the velocity gradients rather than the velocities themselves.

The two analogies we have pointed out differ most obviously in the reverse roles played by scale (wavenumber) and space. However, they differ more essentially in terms of their motivation, the first (with critical phenomena) being more suggestive in physical terms and the second (with field theory) arising from mathematical considerations of the formal renormalization procedure.

In our first analogy, the correlation length in critical phenomena and the dissipation scale in turbulence are intrinsic properties of the system, determined by its detailed dynamical and statistical properties, rather than fixed external inputs. In contrast, the lattice spacing in the first case and the integral scale in the second case are parameters fixed by the experimental setup or the definition of the model. The inverse role of large and small scales in the two cases arises from the different character of "cascade" in the two instances. According to the cascade picture, there is a transfer of excitation on the average from the large turbulent eddies to the small ones by a stepwise process, which is chaotic in nature and entails a loss of memory of the large-scale statistics. Wilson has emphasized [22] that there is also a "cascade of fluctuations" in critical phenomena. Droplet fluctuations nucleated at the lattice-scale in the critical state can grow to the size of the correlation length (and vice versa). However, it is now the details of the lattice structure which are lost and the scale-invariant distributions of the large "droplets" which are universal.

These facts have suggested to several authors [23], 24], [25] that in constructing an RG theory of turbulence, it may be more natural to reverse the usual procedure and to eliminate low-wavenumber shells rather than high-wavenumber 
shells as in the Wilson method. Some cautionary remarks on such a procedure at a physical level are made in 26 . However, our discussion of the field theoretic analogy shows also that to study short-distance UV-scaling, an IRelimination RG is not required. Lattice QCD is a good case in point, where the short-distance asymptotic freedom is studied by the same UV-elimination procedure as used in critical phenomena. The UV-elimination RG is also closely related to an important practical problem of turbulence theory, that of "subgrid-scale eddy modelling," and is naturally described in terms of simple "eddy viscosity" concepts. Indeed, the UV-elimination RG is deeply connected with such eddy viscosity ideas, and RG-invariance under this operation is nothing more than a restatement of the fact that the inertial range theory is independent of the molecular viscosity [20].

Acknowledgements. We would like to thank the participants of the Aspen Workshop on Fully-Developed Turbulence in August 1993 for many useful comments and discussions on the topic of this paper, particularly J. Eggers, M. Nelkin and P. B. Weichman. We thank the last two for suggesting that this work be written up. We thank the Aspen Center for Physics for its hospitality, and the National Science Foundation for its partial support of this work through grant number NSF-DMR-93-14938.

1 W. D. McComb, The Physics of Fluid Turbulence, (Clarendon, Oxford, 1990).

2 M. Nelkin, Phys. Rev. A 9, 388 (1974).

3 P. G. De Gennes, in: Fluctuation, Instability and Phase Transition, (Proc. NATO Adv. Study Inst., Geilo, Norway). T. Riste, ed. (Noordhoff, Leiden), series B (1975), p1.

4 H. A. Rose and P. L. Sulem, J. de Phys. 39, 441 (1978).

$5 \quad$ A. N. Kolmogorov, Dokl. Akad. Nauk. SSSR, 30301 (1941); 3116 (1941); 3199 (1941); 32 16 (1941), Engl. transl. in Proc. Roy. Soc. London A, 4349 (1991).

6 L. D. Landau, Fiz. Zhurn. Sowjetunion 11 26, 545 (1937); English transl. in: D. ter Haar, Men of Physics: L. D. Landau, Vol. II (Pergamon, Oxford, 1969).

7 N. Goldenfeld, Lectures on Phase Transitions and the Renormalization Group, Frontiers in Physics, vol.85. (AddisonWesley, Reading, MA, 1992).

8 G. I. Barenblatt, Similarity, Self-Similarity, and Intermediate Asymptotics (Consultant Bureau, New York, 1979).

9 L. D. Landau and E. M. Lifschitz, Fluid Mechanics, 1st Russian Ed. (Nauka, Moscow, 1944), footnote.

10 U. Frisch and M. Vergassola, Europhys. Lett. 14439 (1991).

11 H. K. Janssen, Z. Phys. B23 377 (1976).

12 C. De Dominicis, J. Phys. (Paris) Colloq. 37113 (1976).

13 F. A. Anselmet et al., J. Fluid Mech. 14063 (1984).

14 A. Vincent and M. Meneguzzi, J. Fluid Mech. 2251 (1991).

15 B. Duplantier and A. W. W. Ludwig, Phys. Rev. Lett. 66247 (1991).

16 G. Eyink, "Multifractals and Lagrangian Field Theory," to appear in a special issue of Chaos, Solitons, and Fractals, Spring 1995.

17 E. Brézin, J. C. Le Guillou and J. Zinn-Justin, Phys. Rev. D 14, 2615 (1976); B 144976 (1976).

18 F. Wegner, in: Localization and Metal-Insulator Transitions. Institute for Amorphous Studies Series, edited by H. Fritzsche and D. Adler (Plenum, New York, 1985).

19 G. L. Eyink, Phys. Lett. A 172, 355 (1993).

20 G. L. Eyink, Phys. Rev. E 48, 1823 (1993).

21 C. W. Van Atta and R. A. Antonia, Phys. Fluids 23252 (1980).

22 K. G. Wilson, Rev. Mod. Phys. 47773 (1975).

23 P. C. Martin and C. de Dominicis, Prog. Theor. Phys. Suppl. 64, 108 (1978).

24 S. Grossmann and E. Schnedler, Z. Phys. B 26, 307 (1977).

25 E. Levich, Phys. Lett. A 79, 171 (1980).

26 R. H. Kraichnan, Phys. Rev. A 25, 3281 (1982). 